\title{
Automatic Identificaiton of Cortical Sulci Using a 3D Probabilistic Atlas
}

\author{
Georges Le Goualher ${ }^{12}$, D. Louis Collins ${ }^{1}$, Christian Barillot ${ }^{2}$, and \\ Alan C. Evans ${ }^{1}$ \\ 1 McConnell Brain Imaging Center, Montréal Neurological Institute, \\ McGill University, Montréal, Canada \\ georges @bic .mni .mcgill. ca, \\ WWW home page: http://ww.bic.mni.mcgill/users/georges \\ ${ }^{2}$ Laboratoire Signaux et Images en Médecine, Université de Rennes, France
}

\begin{abstract}
We present an approach which performs the automatic labeling of the main cortical sulci using a priori information for the 3D spatial distribution of these entities. We have developed a methodology to extract the 3D cortical topography of a particular subject from in vivo observations obtained through MRI. The cortical topography is encoded in a relational graph structure composed of two main features: arcs and vertices. Each vertex contains a parametric surface representing the buried part of a sulcus. Points on this parametric surface are expressed in stereotaxic coordinates (i.e., with respect to a standardized brain coordinate system). Arcs represent the connections between these entities. Manual sulcal labeling is performed by tagging a sulcal surface in the 3-D graph and selecting from a menu of candidate sulcus names. Automatic labeling is dependent on a probabilistic atlas of sulcal anatomy derived from a set of 51 graphs that were labeled by an anatomist. We show how these 3D sulcal spatial distribution maps can be used to perform the identification of the cortical sulci. We focus our attention on the peri-central area (including pre-central, post-central and central sulci). Results show that the use of spatial priors permit automatic identification of the main sulci with a good accuracy.
\end{abstract}

keywords: active model, probabilistic atlas, cerebral cortex, sulci, MRI.

\section{Introduction}

Cortical sulci and gyri define gross anatomical landmarks on the surface of the cerebral cortex. Several studies have suggested that some major functional areas can be located with respect to these anatomical landmarks, allowing $a$ priori localization of functional areas. The most important illustration of such an anatomo-functional correlation comes from the observation that the central sulcus delimits the sensory area (located on its posterior gyrus, known as the postcentral gyrus) from the motor area located on its anterior gyrus (precentral gyrus). Such a priori knowledge of functional localization is incorporated 
in many neurological applications (neurosurgery, for example). However, high inter-individual variability of the cortical topography (associated with the intrinsic complexity of the 3D cortical fold patterns) make the identification of these landmarks a tedious task. We know that variability of the cortical topography is due to gross brain shape differences between individuals, but also to structural deformation of the cortex. This deformation appears in particular when a sulcus (generally associated with a continuous cortical fold) is decomposed in several apparently independent folds on another subject. Similarly, generally connected adjacent sulci can be sometimes disconnected. Finally, tertiary sulci seem to have random patterns. Processes that are used by experts to perform the identification of cortical folds mix both structural and spatial a priori knowledge on the most likely structural patterns of the cortical sulci and their most likely location. Recently, probabilistic brain atlas methodology has been introduced to deal with the intrinsic brain shape variability [1]. This methodology allows the construction of 3D probability maps representing the normal spatial variability of the main anatomical structures. In this paper, we study the use of such a 3D probabilistic atlas of the main sulci, computed from 51 brains, to perform the identification of the cortical folds based on spatial priors.

In the following we describe our methodology to extract a 3D representation of the cortical topography from 3D MRIs. By working systematically in a standardized brain coordinate system, we are allowed to study the 3D spatial variability of the extracted cortical sulci. These probabilistic maps provide us with a priori knowledge on the localization of the main sulci and can be used to assist in the identification of the main cortical sulci. Here, we particularly focus our attention on the identification of the sulci of the peri-central area (precentral, central and postcentral sulcus).

\section{Background}

For several years now, an increasing interest has been given to both the segmentation of the cortical topography as well as its automatic labeling. The range of image processing and pattern recognition methodologies applied to these tasks are wide, leading to a difficult classification or comparison. We have chosen to first make a distinction between methodologies that model the cortical topography of a single subject without labeling individual sulci, from methodologies that attempt to identify specific cortical patterns.

\subsection{Representation of the Cortical Topography}

The goal of the cortical topography modeling is to represent explicitly each cortical fold and the organization of these entities (connection, adjacency). At this step it is particularly convenient to distinguish between :

- modeling of single versus all cortical folds

- modeling of exterior trace versus median surface 
Existing methods can be classed of crossovers between these two subgroups :

- modeling of exterior trace of all cortical folds [2][3][4]

- modeling of the median surface of a cortical fold [5][6]

- modeling of the median surface of all the cortical folds [7] [8] [9]

Considering the fact that more than $2 / 3$ of the total cortical surface is buried within the cortical folds, it is of the utmost importance to model the median surface of the sulci, not only the superior trace. This kind of modeling allows a better understanding of the cerebral cortex topography by showing patterns that previously were only observable form post mortem subjects.

\subsection{Automatic Identification of the Cortical Topography}

Identification of the cortical folds can be proposed through the atlas matching paradigm [10] [2] [4]. In this methodology a brain model (where all anatomical structures have been labeled) is matched to a studied brain. Identification of anatomical structures on the studied brain is then realized by transferring the labels contained in the model to the studied brain through the recovered nonlinear warping. However the considerable inter-subject structural variability of the cerebral cortex topography is not sufficiently well-modeled to allow accurate labeling of individual sulci by this methodology [11]. In particular the basic hypothesis (one to one correspondence between each voxel of the brain model and the studied brain) on which the elastic warping method is based in not verified for the cortical topography.

More complete a priori information about the normal cortical topography is needed to perform a correct identification of the cortical folds. Both statistics about structural patterns of the cortical fold (information which can be seen as being independent from a coordinate system) and information regarding the spatial localization of the cortical folds are necessary to perform a correct identification. In [12] [3] [13] such informations are used to perform the identification of 6 main sulci. Knowledge is expressed in the form of heuristics that are defined for each of the sulci. Recognition is performed as a search through the combination of automatically extracted sulcus patterns that best match a heuristic model (note that heuristics have to be defined for each sulcus of interest). The weakness of this application stems from the fact that the recognition is performed in a sequential manner (recognition of the central sulcus, followed by the research of the pre-central sulcus, ...), a method that seems to differ from the constant accept/reject trial hypothesis as performed by an expert. Another promising application has been developed by Mangin et al. [8]. In this application the cortical topography is modeled as the realization of a random graph. Recognition is performed through graph matching between a model graph containing information about the normal variability of the cortical topography and the corresponding graph of the studied subject brain. The methodology used is based on the structural pattern recognition paradigm where information about the structural decomposition of an object is used to automatically performed its 
identification. While the structural knowledge on the main sulci is explicitly used in this application, spatial priors (which represent also important knowledge to drive the recognition problem) are poorly used. In fact, in this application only a bounding box defines the a priori localization of each sulcus. Moreover, the matching method, based on a simulated annealing algorithm, is computationally intensive and requires the adjustment of numerous ad hoc parameters.

From our knowledge, few methods have been based on the use of spatial priors (however refer to[14]), and of these only a few sample brains are used to estimate the 3D probability maps of the sulci. Also, it is not well known if a good labeling of cortical sulci can be performed through this simple use of spatial priors.

The method we propose here to assist the labeling of the cortical folds consists of assigning to each automatically extracted cortical fold, the sulcus' name for which the associated 3D probability map is maximum, given the location of the actual fold. This involves $i$ ) the extraction of an explicit representation of the cortical topography composed of a set of folds and junctions between these entities; ii) the computation in a standardized 3D stereotaxic space in order to compute the sulcal probability maps and link the actual 3D spatial position of a cortical fold with these computed probability maps. The method we have used to solve i) can be classified as a method which models the median surface of all the cortical folds (refer to section 2.1) and will be described in the following section. The second point (ii) is solved by using an automatic linear matching algorithm which transforms each brain into a standardized stereotaxic space. 3D sulcal distributions are then computed in this common referential frame. Resulting 3D maps (computed from 51 brains) are then used to identify the cortical folds. Results show colored extracted 3D cortical topography graphs, where each color corresponds to a specific sulcus name.

\section{Modeling of the Cortical Topography}

We describe here the method used to extract the cortical topography from a 3D MRI.

\subsection{Preprocessing}

The goal of the preprocessing is to extract the brain tissues, namely the Grey Matter (GM) and the White Matter (WM). An artificial neural network classifier is applied to identify GM/WM/CSF tissue types [15] on 3-D MRI, after correction for 3-D intensity non-uniformity[16]. The classification method uses spatial priors on the location of each tissue type. This last condition implies that all computations must be carried out in common referential frame. Therefore, we use an automatic registration program which uses a 3-D cross-correlation approach to match the single MRI volume with the intensity average of 305 MRI brain volumes previously aligned into standardized stereotaxic space [17]. The registered volumes are then resampled on a $1 \mathrm{~mm}^{3}$ voxel grid. A geometric surface representing the brain cortical surface is automatically extracted using 
iterative minimization of a cost function [18]. This last step separates voxels belonging to the brain from those located in the cerebellum. All following computations are carried out within the standardized stereotaxic space (refer also to section 4).

\subsection{Processing}

Detection of each Sulcus Exterior Trace The method we have used to detect the exterior traces of cortical sulci combines morphological closing (dilatation followed by an erosion) [19] to fill the interspace between adjacent walls of a sulcus and curvature analysis in the cerebral cortex. We have previously shown that the sign of the mean curvature was a good indicator to separate the sulci from the gyri (refer to [20]). By detecting the voxels which have a positive curvature on the bounding hull of the brain we are able to detect the superior traces of the sulci. We apply a thinning method (refer to [21]) in order to reduce this set of voxels to a set of curves and junctions between curves. We obtain at this step the superior traces of the sulci.

Detection of each Sulcus Median Surface We then model each of these curves with our active ribbon method in order to extract the median surface of each sulcus [22].To summarize, the active ribbon method consists of modeling each curve by a spline and subjecting it to a set of forces designed such that the active model will move smoothly from the initial superior trace of the sulcus toward the bottom of the sulcus. The second step of the active ribbon method consists of modeling the successive loci of this active curve, from its initial position towards its final position, by an active surface which converges toward the actual median surface of the fold. The active ribbon method allows for the retrieval of a parametric surface representing the median surface of each fold of the cerebral cortex.

\subsection{Encoding of the Cortical Topography}

The representation of the cortical topography is directly derived from these results :

$$
G_{C T}=\{F, J\}
$$

where $G_{C T}$, the Graph representing the Cortical Topography of a particular subject, is composed of $F$ a set of $f$ folds (one or several folds may constitute a sulcus as defined by an anatomist) and $J$ a set of $j$ junctions (see examples on figure 2).

\section{Computation of $3 \mathrm{D}$ sulcal probabilistic maps}

As said previously, all computations are realized in the same brain-based coordinate system after application of the automatic linear matching algorithm used 
during the preprocessing (refer to section 3.1). The coordinate system is defined as follows :

$-(0, \vec{x}, \vec{y}, \vec{z}):$ where 0 the origin of the coordinate system is the anterior commisure (AC) ; $\vec{x}, \vec{y}, \vec{z}$ are oriented like the brain referential frame defined by Talairach [23], with $\vec{x}$ left-right axis, $\vec{y}$ the postero-anterior axis and $\vec{z}$ the inferior-superior axis.

- $(s x, s y, s z)$ : linear scaling factors. In order to reduce the spatial variability of brain structures due to gross brain size differences, all brains are fit into the same bounding box after registration (refer to [17]).

An anatomist has identified 16 main sulci on 51 extracted cortical topography graphs. These sulci were : (a) the Central Sulcus and (b) the Sylvian Fissure; (c) the Superior, (d) the Middle, and (e) the Inferior Frontal Sulci; (f) the Ascending and $(\mathrm{g})$ the Horizontal Rami of the Sylvian Fissure, as well as (h) the Incisura (located between these other two rami); (i) the Olfactory Sulcus as well as ( $\mathrm{j}$ ) the Sulcus Lateralis, (k) the Sulcus Medialis, (l) the Sulcus Intermedius, and (m) the Sulcus Transversus (all found along the orbitofrontal surface of the brain); (n) the Precentral and (o) the Postcentral Sulci; and finally (p) the Intraparietal Sulcus.

By averaging the labelings of a structure, a continuous 3-D probability field (0.00-1.00 at each voxel) can be constructed for each identified structure. This average is known as a Statistical Probability Anatomy Map (SP_AM)[24].

\section{Sulcus Identification Using a Probabilistic Atlas}

\subsection{Estimation of a Pertinent Probability Threshold}

To define this threshold we study the overlap between adjacent SP_AMs. Illustrations of such overlaps with respect to a selected threshold can be seen on the figure 1.

We conclude that a probability threshold of value $p_{t h r e s h}=0.1$ yields a minimal overlap between adjacent SP_AMs.

\subsection{Identification}

Identification is performed as follows: for each automatically extracted fold we can compute the probability that this fold belongs to each computed SP_AM. We then have a probability vector:

$$
\vec{p}=\left[p\left(\theta_{0}\right), \ldots, p\left(\theta_{m-1}\right)\right]
$$

where $m$ is the number of computed SP_AMs, $\theta_{i}$ is a sulcus name. We then find the maximum element of this vector, let say $p\left(\theta_{k}\right)$, and apply the following rule: 

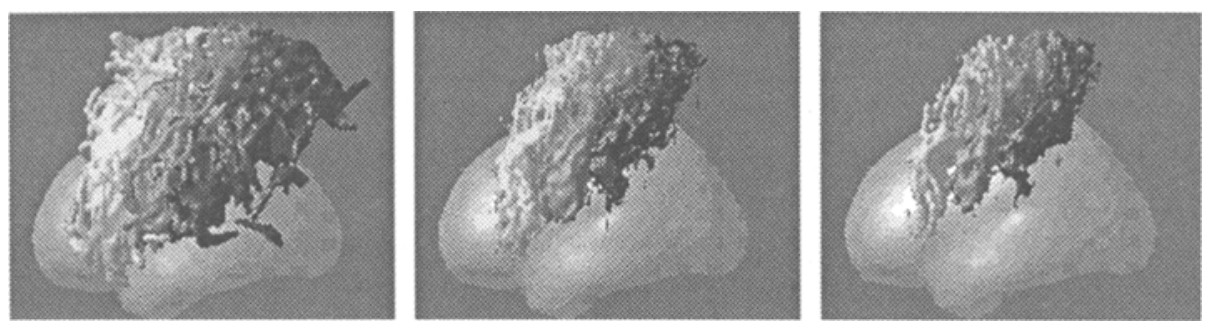

Fig. 1. From left to right : 3D iso-surfaces of the sulcal distribution (computed from 51 subjects) for the precentral, central and postcentral sulci thresholded at different levels. Left image is obtained with a probability threshold $p_{\text {thresh }}=0.001$. All the voxels which have a value superior to this threshold are kept. As the minimum non-zero probability value is $1 / 51$, all non-zero locations of probability maps are kept. One can see the important overlap between these adjacent SP_AMs. Middle: $p_{\text {thresh }}=0.05$. Right: $p_{t h r e s h}=0.1$. One can see the null overlap when taking this threshold. Note that with this threshold, all the voxels kept belong to the same sulcus on at least 6 different subjects upon 51. (note that the brain model, displayed at $50 \%$ of its original size, is only used to figure the orientation)

if $p\left(\theta_{k}\right) \geq p_{t h r e s h}$

then affect the name $\theta_{k}$ to the corresponding fold

else

affect unknown name to the corresponding fold

Results for the identification of 4 brains are shown on figure 2. Almost all parts of superior, middle and inferior frontal sulcus are correctly identified. Only a few small portions of the central, precentral and postcentral sulci are incorrectly identified. This of course has to be interpreted with the caveat that the anatomist's labeling may not be correct for secondary or tertiary sulci.

\section{Conclusion}

The used of spatial priors to identify cortical sulci has been shown to give a very promising initial guess on the labeling of the main cortical sulci. This illustrates an application of the probabilistic atlas paradigm. When extended to all cortical sulci, the use of spatial priors allows to give a vector of initial probabilities for each possible sulcus name for each cortical fold. Even if the simple sulcus name assignment we have presented based on maximum of probability of sulcal spatial distributions will not be always sufficient to give an exact labeling (due to the density and high variability of sulcal pattern), this methodology considerably decreases the state space of the sulcus recognition problem and gives an initialization for a more complex structural probabilistic matching algorithm which could include knowledge on the most likely structural pattern for each cortical 
fold or knowledge on admissible sphape deformations [25]. We are now working on the design of such an algorithm.

\section{Acknowledgments}

The authors are grateful to the Region Council of Brittany (France) for their financial support of this work. They express their appreciation for support from the Canadian Medical Research Council (SP-30), the McDonnell-Pew Cognitive Neuroscience Center Program, the U.S. Human Brain Map Project (HBMP), NIMH and NIDA. This work forms part of a continuing project of the HBMPfunded International Consortium for Brain Mapping (ICBM) to develop a probabilistic atlas of human neuroanatomy.

\section{References}

1. J.C. Mazziota, A.W. Toga, A.C. Evans, P. Fox, and J. Lancaster. A probabilistic atlas of the human brain : theory and rationale for its development. Neuroimage, 2:89-101, 1995.

2. S. SANDOR and R. Leahy. Surface-based labeling of cortical anatomy using a deformable atlas. IEEE Transactions on Medical Imaging, 16(1):41-54, Feb 1997.

3. N.RoyackKers, H. Fawal, M. Desvignes, M. Revenu, and J.M. Travere. Feature extraction for cortical sulci identification. In $9^{\text {th }}$ Scandinavian conference on image analysis, volume 2, pages 110-121, June 1995.

4. G. Subsol, J.P. Thirion, and N. AyACHE. Application of an automatically build 3D morphometric atlas: Study of cerebral ventricle shape. In Karl Heinz Hohne and Ron Kikinis, editors, Visualization in Biomedical Computing (VBC), pages 373-382. LNCS 1131, Sept 1996.

5. G. Le Goualher, C. Barillot, Y. Bizais, and J-M. Scarabin. 3D segmentation of cortical sulci using active models. In SPIE Proceedings of Medical Imaging : Image Processing, volume 2710, pages 254-263, 1996.

6. Marc Vaillant, Christos Davatzikos, and R.Nick Bryan. Finding 3D parametric representations of deep cortical folds. In Workshops on Math. Methods in Biomedical Image Analysis, pages 151-159, 1996.

7. G. Szekely, Ch. Brechuhler, O. Kubler, R. Ogniewickz, and T. Budinger . Mapping the human cerebral cortex using 3-D medial manifolds. In SPIE Visualization in Biomedical Computing, volume 1808, pages 130-144, 1992.

8. J.F.Mangin, J. Regis, I. Bloch, V. Frouin, Y. SAmson, and J. Lopez-Krahe. A MRF based random graph modelling the human cortical topography. In CVRMed, pages 177-183, Nice, France, April 1995. LNCS 905.

9. Gabriele LohmanN, Frithjof KrugGel, and D.Yves von Cramon. Automatic detection of sulcal bottom lines in MR images of the human brain. In Information Processing in Medical Imaging (IPMI), pages 369-374. LNCS 1230, 1997.

10. R. BAJCSY and S. KovACIC. Multiresolution elastic matching. Computer Vision, Graphics, and Image Processing, 46:1-21, 1989.

11. D.L. Collins, G. Le Goualher, R. Venugopal, Z. Caramanos, A.C. Evans, and C. BARILLOT . Cortical constraints for non-linear cortical registration. In K.H. Höene, editor, Visualization in Biomedical Computing, pages 307-316, Hamburg, Sept 1996. 
12. H. Fawall, Contribution à l'étude d'une base de connaissances adaptée da la définition des sillons du cortex cérébral humain. $\mathrm{PhD}$ thesis, Université de Caen, 1995.

13. M. Desvignes, N. Royackkers, H. Fawal, and M. Revenu. Detection and identification of sulci on 3D MRI. In Human Brain Mapping, volume 4, page S410, 1997.

14. F. KRUGGEL. Automatical adaptation of anatomical masks to the neocortex. In Proceedings of the 1th International Conference CVRMed, volume LNCS 905, France, April 1995.

15. A. Zijdenbos, A.C. Evans, F. Riahi, J. Sled, J. Chui, and V. Kollokian. Automatic quantification of multiple sclerosis lesion volume using stereotaxic space. In Visualisation in Biomedical Computing (VBC), pages 439-448. 4th International Conference, LNCS 1131, september 1996.

16. John G. Sled, Alex P. ZIJDEnBos, and Alan C. Evans. A non-parametric method for automatic correction of intensity non-uniformity in MRI data. IEEE Transactions on Medical Imaging, 17(1):87-97, February 1998. submitted December 1996.

17. D.L. Collins, P. Neelin, T.M. Peters, and A.C. Evans. Automatic 3D intersubject registration of $\mathrm{MR}$ volumetric data in standardized Talairach space. Journal of Computer Assisted Tomography, 18(2):192-205, March, April 1994.

18. D.MacDonalD, D. AvIs, and A.C. Evans. Multiple surface identification and matching in Magnetic Resonance Images. In Visualization in Biomedical Computing (VBC), volume 2359, pages 160-169, Rochester, 1994.

19. J. SERRA. Image analysis and mathematical morphology. Academic Press, London, 1982.

20. G. Le Goualher, C. Barillot, L. Le Briquer, and Y. Bizais. 3D detection and representation of cortical sulci. In Computer Assisted Radiology, CAR'95, 1995.

21. G. Malandin, G. Bertrand, and N. Ayache. Topological segmentation of discrete surfaces. In IEEE Conference on Computer Vision and Pattern Recognition, pages 444-449, 1991.

22. G. Le Goualher, C. Barillot, and Y. Bizais. Modeling cortical sulci using active ribbons. International Journal of Pattern Recognition and Artificial Intelligence, 11(8):1295-1315, 1997.

23. J. TAlairach and P. Tournoux. Co-planar stereotactic atlas of the human brain: 3-Dimensional proportional system: an approach to cerebral imaging. Georg Thieme Verlag, Stuttgart, New York, 1988.

24. A.C Evans, D.L. Collins, C. Holmes, T. Paus, D. MacDonald, A. Zijdenbos, A. Toga, P. Fox, J. Lancaster, and J. Mazziota. A 3D probabilistic atlas of normal human neuroanatomy. In Human Brain Mapping, volume 4, page S349, 1997.

25. A. Caunce and C.J. TAYlor. 3D point distribution models of cortical sulci. In ICCV, Bombay, India, january 1998. 

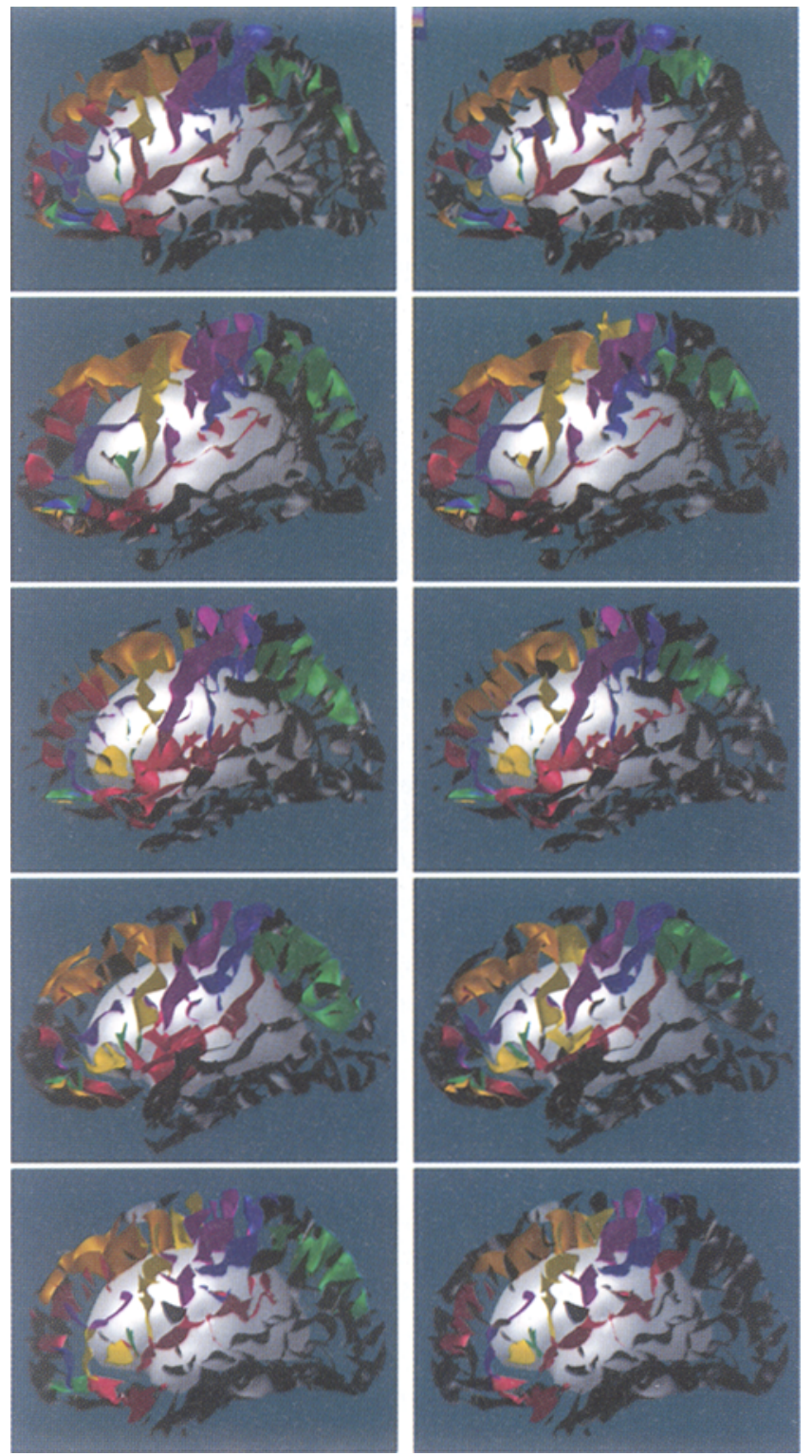

Fig. 2. from top to bottom : 5 different subjects. left : automatic labeling using spatial priors, right : associated manual labeling by an anatomist; magenta : central sul; red : sylvian fiss; frontal lobe : yellow:precentral s.; mustard: superior frontal s.; red : middle frontal s; violet : inferior frontal s.; green : ascending ramus of the syl. fiss; antero-inferior part of the frontal lobe : yellow : horizontal ramus of the syl. fiss.; green : intermedius fronto-orbital s.; blue : lateralis front-orb. sul.; parietal lobe : blue : postcentral s.; green : intraparietal s.; black : unknown; (note that the brain model, displayed at $50 \%$ of its original size, is only used to figure the orientation) 